\title{
Market dynamics immediately before and after financial shocks: Quantifying the Omori, productivity, and Bath laws
}

\author{
Alexander M. Petersen, ${ }^{1}$ Fengzhong Wang, ${ }^{1}$ Shlomo Havlin, ${ }^{2,1}$ and H. Eugene Stanley ${ }^{1}$ \\ ${ }^{1}$ Center for Polymer Studies and Department of Physics, Boston University, Boston, Massachusetts 02215, USA \\ ${ }^{2}$ Minerva Center and Department of Physics, Bar-Ilan University, Ramat-Gan 52900, Israel
}

(Received 10 June 2010; published 27 September 2010)

\begin{abstract}
We study the cascading dynamics immediately before and immediately after 219 market shocks. We define the time of a market shock $T_{c}$ to be the time for which the market volatility $V\left(T_{c}\right)$ has a peak that exceeds a predetermined threshold. The cascade of high volatility "aftershocks" triggered by the "main shock" is quantitatively similar to earthquakes and solar flares, which have been described by three empirical laws-the Omori law, the productivity law, and the Bath law. We analyze the most traded 531 stocks in U.S. markets during the $2 \mathrm{yr}$ period of 2001-2002 at the $1 \mathrm{~min}$ time resolution. We find quantitative relations between the main shock magnitude $M \equiv \log _{10} V\left(T_{c}\right)$ and the parameters quantifying the decay of volatility aftershocks as well as the volatility preshocks. We also find that stocks with larger trading activity react more strongly and more quickly to market shocks than stocks with smaller trading activity. Our findings characterize the typical volatility response conditional on $M$, both at the market and the individual stock scale. We argue that there is potential utility in these three statistical quantitative relations with applications in option pricing and volatility trading.
\end{abstract}

DOI: 10.1103/PhysRevE.82.036114

PACS number(s): 89.65.Gh, 91.30.Px, 89.75.Da, 05.45.Tp

\section{INTRODUCTION}

Financial fluctuations have been a topic of study for economists [1,2], mathematicians [3], and physicists [4-9]. Here, we study financial fluctuations using concepts developed in the field of seismology $[10,11]$ and analogies from turbulent dynamics in our description of market main shock magnitudes in order to analyze the dynamic response of markets to financial shocks. We identify parallels between energy cascades and information cascades, and also between turbulent bursts and the clustering of volatility [12]. Our results demonstrate three statistical regularities which relate the volatility magnitude $M \equiv \log _{10} V\left(T_{c}\right)$ to the market response before and after market shocks.

Common financial "shocks" are relatively smaller in the volatility magnitude, the duration, and the number of stocks affected than the extremely large and infrequent financial crashes. Devastating financial shocks such as Black Monday (October 20, 1987) have significant aftershocks that can last for several months, and this "dynamic relaxation" is similar to the aftershock cascade following an earthquake [13]. Here, we aim to better understand market shocks over a range of $M$ values. While the previous studies have focused on at most a few large crashes, we use a large data set of 219 financial main shocks observed in American markets over the $2 \mathrm{yr}$ period of 2001-2002. We analyze 531 frequently traded stocks corresponding to approximately 44000000 volatility records at a 1 min time resolution. We find three quantitative relations which enable answering such questions as:

(i) How does the rate of volatility aftershocks decay with time, and how do the decay parameters relate to the main shock magnitude $M$ ?

(ii) How many aftershocks above a given threshold can be expected after a main shock of magnitude $M$ ?

(iii) What is the relation between the value of the main shock volatility $V\left(T_{c}\right)$ and the second largest aftershock (or preshock)?
These three questions have been studied for geophysical earthquakes, and the corresponding statistical laws are referred to, respectively, as the Omori law, the productivity law, and the Bath law. The Omori law was first investigated in the context of financial crashes by Lillo and Mantegna [13], who found a power-law relaxation of fluctuations at a 1 min time resolution for the S\&P500 over the 100 day period following the Black Monday crash. Power-law relaxation of aftershocks is also observed for long periods following several other medium-size crashes [14], and also for short periods up to several days following U.S. Federal Reserve interest rate change announcements [15]. One key feature of long-range relaxation dynamics is the scale-free decay of large fluctuations that is typical of a system with memory, and which is complemented by self-similarity in the decay substructure [14].

We find similar perturbation-response dynamics in the intraday volatility (absolute return) time series for many single stocks on numerous days, indicating that markets respond in a common way to perturbations that range in size from everyday market fluctuations to infrequent market crashes. Interestingly, the market is very responsive to Federal Open Market Committee (FOMC) news, either in the form of subtle hints from the Fed or actual rate changes (expected or unexpected), because Fed Target rates serve as a benchmark and barometer for both U.S. and world markets [15]. Methods have been developed to use the interplay between the U.S. Treasury Bill and the Federal Funds effective rate in order to estimate the future movement of the Federal Funds target rate [16]. More complex methods to estimate the probability of interest rate change involves analyzing the price movement of expiring derivative contracts [17]. The connection between macroeconomic factors and financial markets is a tribute to the complexity and connectivity of economic systems. It is a further indicator that news, in addition to complex order-book dynamics, can play a significant role in 
explaining the large rate of occurrence of large fluctuations in markets.

Here, we quantify the rate $n\left(\left|t-T_{c}\right|\right)$ of volatility shocks at time $t$ both before and after a market shock occurring at time $T_{c}$. In order to determine $T_{c}$, we develop a method for selecting a critical time $T_{c}$ from a set of candidate times $\left\{t_{c}\right\}$ for which the collective market volatility of $S$ individual stocks is above a given threshold. For 19 particular dates corresponding to days with FOMC announcements, we compare the values of calculated $T_{c}$ with the reported values of $T$ analyzed in [15], and we find good prediction of $T$ using this method. After this calibration, we study the relaxation dynamics of $S=531$ stocks, analyzing the Omori law, the productivity law, and the Bath law for the dynamics both before $\left(t<T_{c}\right)$ and after $\left(t>T_{c}\right)$ the main market shock.

In Sec. II we discuss the data, the quantitative methods used to calculate $n\left(\left|t-T_{c}\right|\right)$, and define collective market movement. In Sec. III A we quantify the threshold for selecting candidate cascades and calibrate using known values of $T_{c}$ corresponding to FOMC meetings. In Sec. III B we describe the method for choosing $T_{c}$ from each significant cascade we identify. In Sec. IV we discuss the Omori-law parameters $\alpha$ and $\Omega$, the productivity parameter $\Pi$, and the Bath law parameter $B$. We note that both $\Pi$ and $B$ are independent of the dynamical model, and hence do not depend on $n\left(\left|t-T_{c}\right|\right)$, the functional form of the relaxation dynamics. For each of the statistical laws, we compare the results we obtain for the market average with the results we obtain for individual stocks.

\section{DATA ANALYZED}

For the 2 yr period of 2001-2002, we analyze trades and quotes data of more than 500 stocks listed on the NASDAQ and New York Stock Exchange. In order to analyze the most important subset of stocks, we rank each stock by the average number of transactions per minute. We find $S=531$ stocks with an average of more than three transactions per minute, $S=136$ stocks with an average of more than ten transactions per minute, and $S=20$ stocks with an average of more than 50 transactions per minute. Unless otherwise stated, our results correspond to the top $S=531$ stocks, but all results become more statistically significant for smaller subsets of more heavily traded (bell weather) stocks.

In this paper, we study the volatility $v_{j}(t)$ of the intraday price time series $p_{j}(t)$ for stock $j$. The intraday volatility (absolute returns) is expressed as

$$
v_{j}(t) \equiv\left|\ln \left[p_{j}(t) / p_{j}(t-\delta t)\right]\right|,
$$

where we choose here $\delta t=1 \mathrm{~min}$, so that we can analyze the dynamics immediately before and immediately after market shocks. To compare stocks, we scale each volatility time series by the standard deviation over the entire period analyzed. We then remove the "U-shaped" intraday trading pattern (averaged over 531 stocks) from each time series. This establishes a normalized volatility in units of standard deviation for all minutes during the day and for all stocks analyzed (see Ref. [14]).

We introduce a volatility threshold $q$ which defines a binary volatility time series $n_{j}(t)$ for each stock $j$, which we calculate from the normalized volatility time series $v_{j}(t)$ as

$$
n_{j}(t) \equiv \begin{cases}1, & v_{j}(t) \geq q \\ 0, & v_{j}(t)<q .\end{cases}
$$

We find that a volatility threshold $q \equiv 3 \sigma$ is large enough to distinguish between significant fluctuations and normal background activity. We also choose this value $q \equiv 3 \sigma$ to provide comparison with the analysis performed in [15]. The rate $n(t)$ measures the fraction of the market exceeding $q$ at time $t$,

$$
n(t) \equiv \frac{1}{S} \sum_{j=1}^{S} n_{j}(t) .
$$

The rate $n_{j}(t)$ quantifying the volatility of a single stock $j$ corresponds to the limit $S \rightarrow 1$. We define the average market volatility $V(t)$ similarly by

$$
V(t) \equiv \frac{1}{S} \sum_{j=1}^{S} v_{j}(t)
$$

A market shock at time $T_{c}$ may result from exogenous (external) news or endogenous herding $[18,19]$. In many cases, the market shocks can be linked to exogenous news using archived news feeds that cover and summarize daily market events [20]. In order to analyze market dynamics symmetrically around a market shock at time $T_{c}$, we analyze the per unit time rate $n\left(\left|t-T_{c}\right|\right)$ around the time $T_{c}$. It has been empirically observed that the response dynamics in financial markets show a power-law decay [13-15,21-23],

$$
n\left(\left|t-T_{c}\right|\right) \sim \alpha\left|t-T_{c}\right|^{-\Omega},
$$

where $\Omega$ is called the Omori power-law exponent, $\alpha$ is the cascade amplitude, $t<T_{c}$ corresponds to before the main shock, and $t>T_{c}$ corresponds to after the main shock. For comparison, $n\left(\left|t-T_{c}\right|\right)$ is constant for stochastic processes with no memory, corresponding to $\Omega \equiv 0$. Hence, for an empirical value $\Omega \approx 0$, the rate $n\left(\left|t-T_{c}\right|\right)$ is indistinguishable from an exponential decay for $\left|t-T_{c}\right|<\bar{t}$, where $\bar{t}$ is the characteristic exponential time scale. However, for larger values of $\Omega$, the exponential and power-law response curves are distinguishable, especially if several orders of magnitude in $\tau$ are available.

Instead of analyzing $n\left(\left|t-T_{c}\right|\right)$, we perform our quantitative analysis on $N\left(\left|t-T_{c}\right|\right)$, the cumulative number of events above threshold $q$ at time $t \mathrm{~min}$, where by definition

$$
N\left(\left|t-T_{c}\right|\right)=\int_{T_{c}}^{t} n\left(\left|t^{\prime}-T_{c}\right|\right) d t^{\prime} \sim \beta\left(\left|t-T_{c}\right|\right)^{1-\Omega},
$$

for market co-movement, and

$$
N^{j}\left(\left|t-T_{c}\right|\right)=\int_{T_{c}}^{t} n_{j}\left(\left|t^{\prime}-T_{c}\right|\right) d t^{\prime} \sim \beta_{j}\left(\left|t-T_{c}\right|\right)^{1-\Omega_{j}},
$$

for the activity of stock $j$. We perform our regression analysis on $N^{j}\left(\left|t-T_{c}\right|\right)$ because it is less noisy and monotonic as compared to $n_{j}\left(\left|t-T_{c}\right|\right)$.

Hence, for a given day, we calculate the cumulative time series $N^{j}(t)$ from $n_{j}(t)$ for each stock $j$, where $t=0$ corre- 


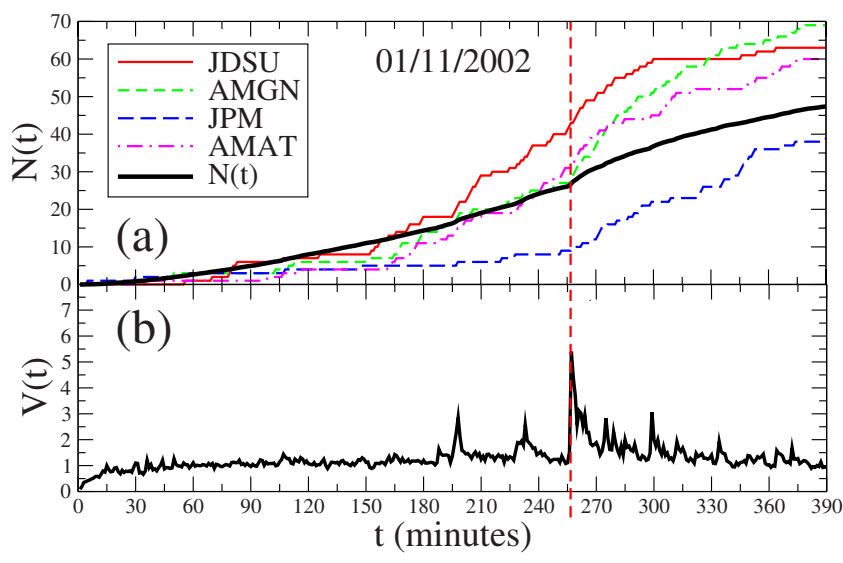

FIG. 1. (Color online) Typical volatility curves on 01/11/2002 with market shock at $T_{c}=256 \mathrm{~min}$. (a) The cumulative volatility $N^{j}(t)$ for the stock of several large companies has varying behavior before $T_{c}$, but each stock shown begins to cascade soon after $T_{c}$. The market average $N(t)$ over all $S=531$ stocks analyzed demonstrates a distinct change in curvature at $t=T_{c}$. (b) The average market volatility $V(t)$ demonstrates a sharp peak at $T_{c}$, and also two precursor events at $t \approx 190$ and $\approx 230 \mathrm{~min}$.

sponds to the opening bell at 9:30 a.m. eastern time (ET). For comparison, we also analyze the average market response $N(t)$ of the $S$ stocks under consideration, which complements the study of individual stocks.

To demonstrate our approach, in Fig. 1 we plot $V(t), N(t)$, and also $N^{j}(t)$ for four single stocks on $01 / 11 / 2002$, a day when there was a large market shock corresponding to a publicized comment by the Fed chairman Alan Greenspan concerning economic recovery which occurred at approximately $T_{c}=255 \mathrm{~min}$ after the opening bell.

In order to compare the dynamics before and after the market shock, we first separate the intraday time series $N(t)$ into two time series $N_{b}\left(t \mid t<T_{c}\right)$ and $N_{a}\left(t \mid t>T_{c}\right)$. Then, to treat the dynamics symmetrically around $T_{c}$, we define the displaced time $\tau=\left|t-T_{c}\right| \geq 1$ as the temporal distance from $T_{c}$. As an illustration, we plot in Fig. 2 the time series on $01 / 11 / 2002$ as a function of $\tau$. We then employ a linear fit to find the $\tau$ dependence of both $N_{b}(\tau) \equiv N\left(T_{c}\right)-N\left(\left|t-T_{c}\right|\right)$ and $N_{a}(\tau) \equiv N\left(t-T_{c}\right)-N\left(T_{c}\right)$ on a log-log scale to estimate the Omori power-law exponents $\Omega_{b}$ before the news and $\Omega_{a}$ after the news. By analogy, we define $\alpha$ to be the amplitude $\alpha=\beta(1-\Omega)$ before $T_{c}$ as $\alpha_{b}$ and after the shock as $\alpha_{a}$.

\section{METHOD FOR DETERMINING $T_{c}$}

\section{A. Calibration using FOMC announcements}

We use $n(t)$ to quantitatively determine times $T_{c}$ in which the market is moving together, possibly in response to an external market shock or possibly as a result of endogenous herding. In Fig. 3 we plot the average daily pattern for $\bar{n}(t)$ and the standard deviation $\sigma(t)$. The values of $\bar{n}(t)$ and $\sigma(t)$ are not stationary, so we remove the daily trend from $n(t)$ by defining the detrended quantity $n^{\prime}(t) \equiv[n(t)-\bar{n}(t)] / \sigma(t)$. In order to distinguish significant moments of market comovement from background fluctuations, we use a significance
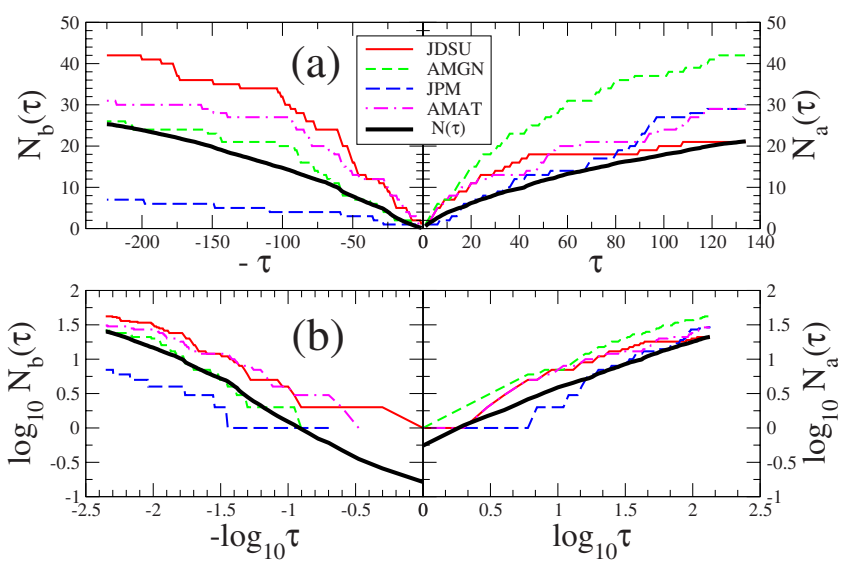

FIG. 2. (Color online) (a) An illustration of $N_{b}(\tau)$ and $N_{a}(\tau)$ for the same set of curves plotted in Fig. 1. The displaced time $\tau=\left|t-T_{c}\right|$ is defined symmetrically around $T_{c}=256 \mathrm{~min}$ on $01 / 11 /$ 2002. (b) $\log _{10} N_{b}(\tau)$ and $\log _{10} N_{a}(\tau)$ are linear with $\log _{10} \tau$ over two orders of magnitude on a logarithmic scale. The Omori parameters in Eq. (5) calculated from $N(t)$ are $\Omega_{b}=0.09 \pm 0.01$, $\alpha_{b}=0.21 \pm 0.01$ and $\Omega_{a}=0.32 \pm 0.01, \alpha_{a}=0.81 \pm 0.01$.

threshold which we obtained from the distribution of market activity over the entire data set analyzed. Hence, we analyze the quantity $x(t)$ defined as

$$
x(t) \equiv n(t) \frac{n(t)-\bar{n}(t)}{\sigma(t)},
$$

which is the product of $n(t)$ and $n^{\prime}(t)$. The value of $n(t)$ quantifies the size of the market comovement, while $n^{\prime}(t)$ quantifies the significance of the market comovement. Be-

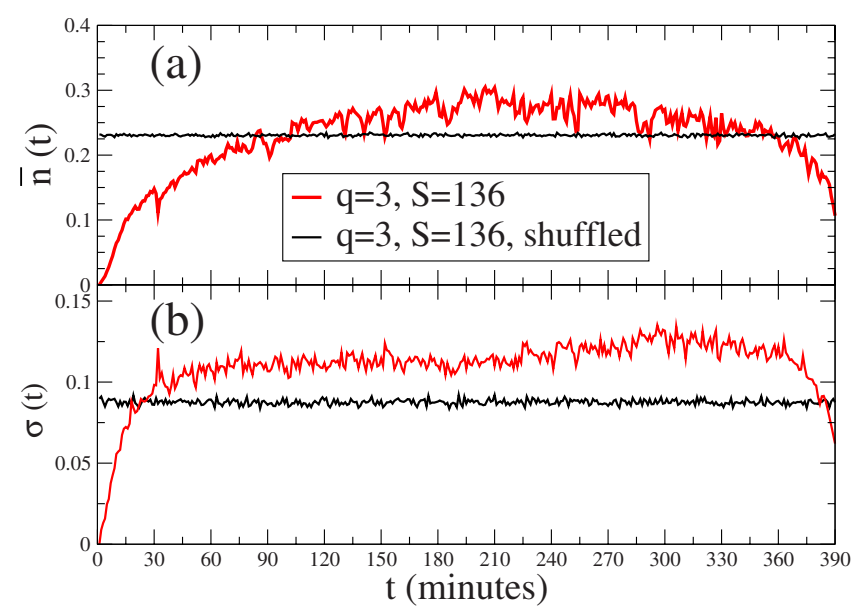

FIG. 3. (Color online) The fraction $n(t)$ of the market above the volatility threshold $q$ is nonstationary through the trading day. We plot in (a) the average daily trading pattern $\bar{n}(t)$ for $S=136$ stocks and in (b) the corresponding standard deviation to demonstrate the trends we remove in the normalized quantity $n^{\prime}(t)$. In practice, we use the smoothed average of these curves in order to diminish statistical fluctuations on the minute-to-minute scale. For comparison, we compute $\bar{n}_{s h}(t) \approx 0.23$ and $\sigma_{s h} \approx 0.09$ for shuffled $v_{i}(t)$. The values of $\bar{n}(t)$ provide an estimate for the background market comovement that can be attributed to random fluctuations. 


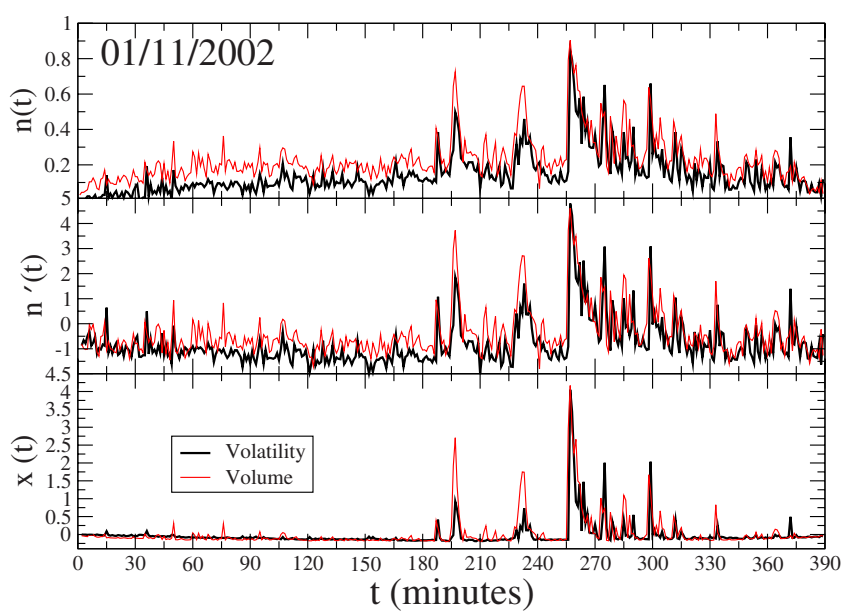

FIG. 4. (Color online) Example of market comovement $n(t)$ in both price volatility and total volume, and the qualitative relationship between the quantities $n(t), n^{\prime}(t) \equiv[n(t)-\bar{n}(t)] / \sigma(t)$, and $x(t)$ $\equiv n(t) n^{\prime}(t)$. The market shock on $01 / 11 / 2002$ occurred at $T_{c}=256$ in response to a public comment by the Fed chairman A. Greenspan concerning economic recovery [20].

cause $\bar{n}(t)$ is not constant during the day, we consider the normalized quantity $n^{\prime}(t)$ in order to remove the intraday pattern. Then, to restrict our analysis to relatively large market comovements, we eliminate times toward the beginning and end of each day, when average market activity is lower (significant morning activity is often related to overnight news [24]). We analyze the quantity $x(t)$, which is large only if both $n(t)$ and $n^{\prime}(t)$ are large. Figure 4 demonstrates how the quantity $x(t)$ is useful for amplifying market comovement and provides an illustration of a significant shock with substantial preshock and aftershock dynamics.

We analyze the time series $x(t)$ in order to select the set of times $\{t\}$ of the market shocks that are large in the fraction of the market involved [large $n(t)]$ as well as significant with respect to the time in which they occur [large $\left.n^{\prime}(t)\right]$. We determine a significance threshold $x_{c}$ from the probability density function (pdf) of $x(t)$ as in Fig. 5. As a null model, we shuffle the order of each intraday time series $v_{j}(t)$ and obtain a shuffled market volatility rate $n_{s h}(t)$ for each day. This preserves the empirical pdf of $v_{j}(t)$ but removes the correlations that exist in the temporal structure of $v_{j}(t)$. We also plot $\overline{n_{s h}}(t) \approx 0.23$ in Fig. 3 which corresponds to a residual 0.23 comovement due to random fluctuations. We compare the pdf's for $x(t)$ and $x_{s h}(t)$ in Fig. 5(b) and observe a significant divergence for $x(t)>1$.

We calibrate our method for determining $T_{c}$ from candidate cascades by using the known reported values $T$ corresponding to Fed announcements. We choose the value $x_{c}$ $=1.0$ which reproduces with the best accuracy the values of $T$ that we provide for comparison in Table I. The value of $x_{c}$ $=1.0$ results in $5804 \mathrm{~min}$ out of $190000 \mathrm{~min}$ analyzed for which $x(t)>x_{c}$, or roughly $3 \%$ of the 2 yr period with significant market comovement.

\section{B. Determining $T_{c}$ from candidate cascades}

In a typical trading day there are many large fluctuations for both individual stocks and indices such as the S\&P 500
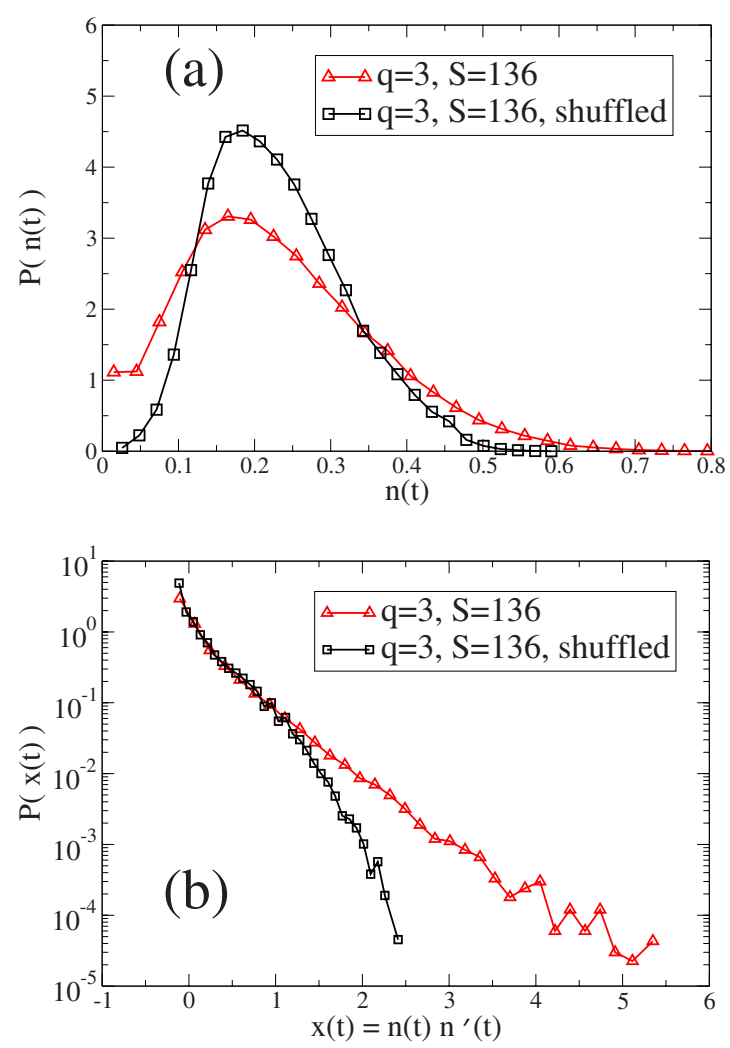

FIG. 5. (Color online) Using the volatility threshold $q=3$ and $S=136$ stocks, we determine the market comovement threshold $x_{c}$ from the pdf of $x(t) \equiv n(t) n^{\prime}(t)$. (a) The pdf for the $190000 \mathrm{~min}$ analyzed of the volatility rate $n(t)$ corresponding to the fraction of the market with volatility $v_{i}(t)>q$. (b) The pdf of $x(t)$, where in the quantity $x(t)$ we have removed the average daily trend of $n(t)$, so that $x(t)$ is relatively large when market comovement is large and significant. For comparison, we also plot the pdf of $x_{s h}(t)$ computed from randomly shuffled volatility time series $v_{i}(t)$. We find a divergence between the pdf's of $x(t)$ and of $x_{s h}(t)$ for $x>1.0$, which we define as the comovement threshold $x_{c} \equiv 1$ in our analysis.

and DOW. Analysis of the return intervals and the cross correlations in financial time series shows that significant statistical regularities exist [25-31]. This fact is evident in the robust probability density function of volatility which has a stable power-law tail for a wide range of time scales ranging from 1 min to several days $[4,7,33,34]$. We select market cascades that are above a "spurious fluctuation" threshold, which we define by randomizing the order $v_{i}(t)$. We use the corresponding shuffled values $n_{s h}(t)$ as a proxy for background noise.

We find on average approximately $12 \mathrm{~min}$ per day above the threshold $x_{c} \equiv 1.0$. So here we develop a method for selecting the most likely time $T_{c}$ from all candidate times with $x(t)>x_{c}$. For a given day, we collect all values of $x(t)>x_{c}$ into a subset $\left\{x^{\prime}(t)\right\}$ of size $z$. From this subset, we divide the $z$ values into $k$ cascades $\left\{x^{\prime}(t)\right\}_{i}$, which we define as localized groups using the criterion that a cascade ends when the time between the last $x^{\prime}$ in cascade $i$ is separated from the first $x^{\prime}$ in cascade $i+1$ by a time window greater than $\Delta l \min$. We next assign to each cascade group $\left\{x^{\prime}(t)\right\}_{i}$ a weight equal to the sum of the $x^{\prime}(t)$ values belonging to the given cascade 
TABLE I. Comparison of announcement times $T$ (as reported in New York Times) with the market clustering times $T_{c}$ calculated using a threshold $x_{c}=1.0$, cascade window $\Delta l \equiv 60 \mathrm{~min}$, and $S$ $=136$ stocks. The value of $x\left(T_{c}\right)$ corresponds to the largest value out of all the candidates $\{x\}$ in the most significant cascade of the particular day. Dates of 19 FOMC meetings in the $2 \mathrm{yr}$ period between January 2001 and December 2002, where the Federal Funds Target rate $\left(R_{\text {new }}\right)$ was implemented by the rate change $(\Delta R)$ at $(T)$ minutes after the opening bell at 9:30 a.m. ET [32]. The absolute relative change $\left|\Delta R / R_{\text {old }}\right| \equiv|\Delta R(t) / R(t-1)|$ has typically filled the range between 0.0 and 0.25 . Note: Date** refers to unscheduled meetings, in which the announcement time did not correspond to 2:15 p.m. ET $(T=285 \mathrm{~min})$.

\begin{tabular}{cllcrr}
\hline \hline FOMC date & $\begin{array}{c}R_{\text {new }} \\
(\%)\end{array}$ & $\Delta R$ & $\frac{\Delta R}{R_{\text {old }}}$ & \multicolumn{1}{c}{$T$} & $T_{c}$ \\
\hline $01 / 03 / 01^{* *}$ & 6 & -0.5 & -0.077 & 210 & 227 \\
$01 / 31 / 01$ & 5.5 & -0.5 & -0.083 & 285 & 290 \\
$03 / 20 / 01$ & 5 & -0.5 & -0.091 & 285 & 286 \\
$04 / 18 / 01^{* *}$ & 4.5 & -0.5 & -0.100 & 90 & 88 \\
$05 / 15 / 01$ & 4 & -0.5 & -0.111 & 285 & 287 \\
$06 / 27 / 01$ & 3.75 & -0.25 & -0.063 & 285 & 285 \\
$08 / 21 / 01$ & 3.5 & -0.25 & -0.067 & 285 & 286 \\
$09 / 17 / 01^{* *}$ & 3 & -0.5 & -0.143 & 0 & 16 \\
$10 / 02 / 01$ & 2.5 & -0.5 & -0.167 & 285 & 288 \\
$11 / 06 / 01$ & 2 & -0.5 & -0.200 & 285 & 292 \\
$12 / 11 / 01$ & 1.75 & -0.25 & -0.125 & 285 & 287 \\
$01 / 30 / 02$ & 1.75 & 0 & 0.00 & 285 & 289 \\
$03 / 19 / 02$ & 1.75 & 0 & 0.00 & 285 & 293 \\
$05 / 07 / 02$ & 1.75 & 0 & 0.00 & 285 & 287 \\
$06 / 26 / 02$ & 1.75 & 0 & 0.00 & 285 & 286 \\
$08 / 13 / 02$ & 1.75 & 0 & 0.00 & 285 & 291 \\
$09 / 24 / 02$ & 1.75 & 0 & 0.00 & 285 & 291 \\
$11 / 06 / 02$ & 1.25 & -0.5 & -0.286 & 285 & 286 \\
$12 / 10 / 02$ & 1.25 & 0 & 0.00 & 285 & 295 \\
\hline \hline
\end{tabular}

group, and we select the cascade group with the largest weight as the most significant cascade. Within the most significant cascade group, we choose the time corresponding to the maximum value of $x^{\prime}(t)$ as the time $T_{c}$ of the main shock. We calibrate this method using the reported times for the 19 FOMC interest rate meeting announcements and find that the values $\Delta l \equiv 60$ and $x_{c}=1.0$ best reproduce the known set $\{T\}$, which we provide for comparison in Table I.

Using the parameter values $x_{c}=1.0$ and $\Delta l \equiv 60$, we find 373 days with market shocks out of 495 days studied. If the values of $x^{\prime}(t)$ were distributed uniformly across all days, then the probability of finding 122 days without one $x^{\prime}(t)$ is vanishingly small, which confirms that $x^{\prime}(t)$ 's group together forming cascades. We remove all days where $T_{c}$ is within $\Delta t \equiv 90$ min of opening $(t=0)$ or closing $(t=390)$, and all $T_{c}$ 's that occur on half-days (days before or after the 4 th of July, Thanksgiving, or Christmas), resulting in the data set $\left\{T_{c}\right\}$ constituting 219 individual days. We also analyzed the subset of 156 market shocks within $\Delta t \equiv 120$ min of opening or closing and find analogous results as those reported here for $\Delta t \equiv 90$.
Furthermore, in order to test the dependence of the data set $\left\{T_{c}^{(1)}\right\}$ found for the time resolution $\delta t=1 \mathrm{~min}$ used in this paper, we also compare the values of $\left\{T_{c}^{(5)}\right\}$ and $\left\{T_{c}^{(10)}\right\}$ found using a volatility series with $\delta t=5 \mathrm{~min}$ and $\delta t=10 \mathrm{~min}$ resolutions, respectively [see Eq. (1)]. For each of the 219 days with a $T_{c}$ value we calculate the absolute difference in the time value $T_{c}^{(\delta t)}$ using two values of $\delta t$. We use similar values of $x_{c}$ for each time resolution, so that the numbers of days with market shocks for each resolution are approximately equal. The difference in $T_{c}^{(\delta t)}$ depends on the resolution $\delta t$ and the locality $\delta T_{c}$ associated with each market shock. The averages of the absolute differences for three values of $\delta t$ are $\left|T_{c}^{(5)}-T_{c}^{(1)}\right|=9 \mathrm{~min}$ and $\left|T_{c}^{(10)}-T_{c}^{(1)}\right|=15 \mathrm{~min}$. We estimate the standard error for a particular time resolution $\delta T_{c}^{(\delta t)} \approx 2 \delta t$, which implies that $\delta T_{c}^{(1)} \approx 2 \mathrm{~min}$ for the $1 \mathrm{~min}$ time resolution. Hence, the use of $T_{c} \pm \delta T_{c}$ does not significantly change the results of this paper. In the next section, we analyze the empirical laws that quantify the response dynamics both before and after significant market shocks.

\section{RESULTS}

The analysis performed in this paper is largely inspired by the analogies between financial market crashes and earthquakes. Here, we identify 219 cascades that meet our volatility significance criterion and that are within $\Delta t$ $=90 \mathrm{~min}$ of the opening or closing of the trading day. For this set of shocks, we analyze the dynamics over the $90 \mathrm{~min}$ period immediately before $T_{c}$ and the $90 \mathrm{~min}$ period $\mathrm{im}$ mediately after $T_{c}$ using the framework developed in earthquake research [35-41]. Although we present results for only $\Delta t=90$, we also analyzed the subset of 156 shocks within $\Delta t=120 \mathrm{~min}$ of the beginning and ending of the trading day and found analogous results. We restrict our analysis to the local time period $2 \Delta t$ within the trading day, so that our results are minimally affected by overnight effects and overlapping shocks (since the frequency of shocks larger than our threshold $x_{c}$ used here is approximately one per trading day). Hence, our analysis of the size dependence of market relaxation dynamics, where we relate the cascade parameters to the market shock magnitude $M \equiv \log _{10} V\left(T_{c}\right)$, pertains to the intraday horizon of market shocks which occur relatively frequently.

A recent study finds significant evidence of Omori powerlaw relaxation both before and after common FOMC interest rate announcements [15] and uses the relationship between the overnight effective rate and the U.S. 6-month Treasury Bill to estimate the magnitude of the financial news shock. The dynamics before the Fed announcements, which are regularly scheduled and preannounced, are consistent with market's anticipated surprise in the Fed news, while the dynamics after the announcements are related to the market's perceived surprise in the Fed news. The Federal Reserve uses the "announcement effect" [42] to control the federal funds market. Despite the calculated monetary policy, the markets react quite strongly to the news, with large Omori-law relaxation cascades that typically correspond to the relatively large $M$ found in this paper.

Closely related to the Omori relaxation of aftershocks is the productivity law, which establishes a power-law relation- 

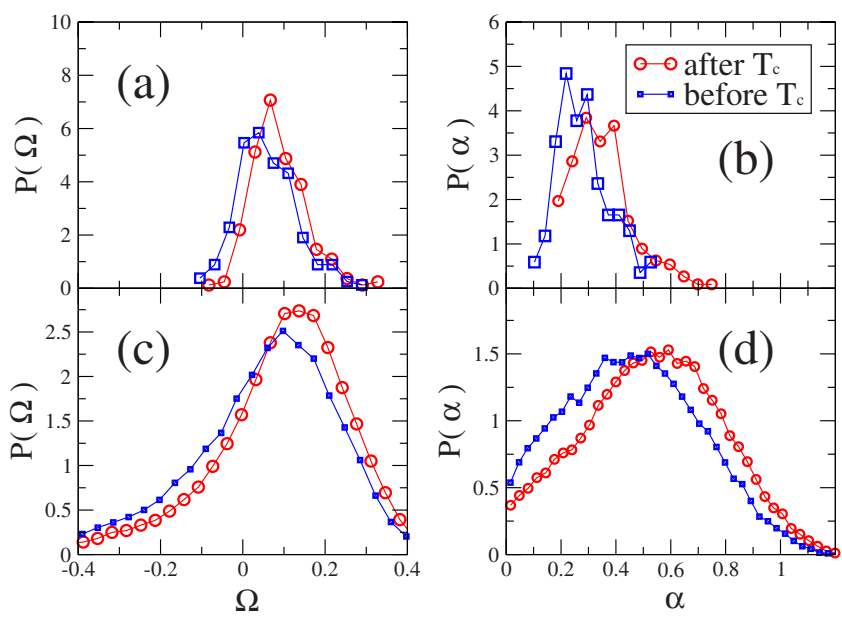

FIG. 6. (Color online) (a) and (b) Comparison of the probability density functions $P(\alpha)$ and $P(\Omega)$ of Omori parameters $\alpha$ and $\Omega$ computed from the average market response $N_{a, b}(\tau)$. (c) and (d) The analogous pdf plots computed from individual stock response $N_{a, b}^{j}(\tau)$. The average and standard deviations of each data set are (a) $\Omega_{a}=0.09 \pm 0.07, \Omega_{b}=0.06 \pm 0.07$; (b) $\alpha_{a}=0.35 \pm 0.11$, $\alpha_{b}=0.28 \pm 0.09$; (c) $\Omega_{a}=0.08 \pm 0.20, \Omega_{b}=0.03 \pm 0.22$; and (d) $\alpha_{a}=0.53 \pm 0.25, \alpha_{b}=0.46 \pm 0.24$. Values of both $\Omega_{a}$ and $\alpha_{a}$ are consistently larger than $\Omega_{b}$ and $\alpha_{b}$, indicating that the response time after $T_{c}$ is shorter than the activation time leading into $T_{c}$. However the response cascade after $T_{c}$ has, generally, a larger amplitude.

ship between the numbers of aftershocks or preshocks that follow or precede a main shock of magnitude $M$. This is analogous to earthquake analysis where the magnitude is defined as $M \approx(2 / 3) \log _{10} E$ [39], where $E$ is the energy associated with the stress released by the main shock. We justify our analogy between market volatility $V$ and earthquake energy $E$ by comparing the cumulative distribution,

$$
P(V>s) \sim s^{-\eta_{V}},
$$

of volatility in financial markets with the cumulative distribution,

$$
P(E>s) \sim s^{-\eta_{E}},
$$

of energy $E$ in seismic earthquakes. Both cumulative distributions are asymptotically power laws, with $\eta_{V} \approx 3[33,34]$ and the Gutenberg-Richter law $\eta_{E} \approx 2 / 3$ [38].

For the set of 219 market shocks we analyze, we find a wide range of $V\left(T_{c}\right)$, and hence a wide range of cascade dynamics. We analyze the dynamics only within the trading day, so that we can be confident that the dynamics after and before $T_{c}$ are related to the market shock $V\left(T_{c}\right)$. Also, for relatively small $V\left(T_{c}\right)$, it may be difficult to distinguish cascade preshocks (aftershocks) from opening and closing effects and volatility resulting from overnight news. Furthermore, we only analyze the first $\Delta t \equiv 90 \mathrm{~min}$ of each $N_{a}(\tau)$ and $N_{b}(\tau)$ time series, so that the comparison of productivity $P_{a, b}(\Delta t)$ is not affected by time series of variable length.

In Fig. 6 we plot the pdf of Omori parameter values $\Omega_{a, b}$ and $\alpha_{a, b}$ obtained from the power-law fits of $N_{b}(\tau)$ and $N_{a}(\tau)$. Figures 6(a) and 6(b) show the distribution of parameter values calculated for the average market responses $N_{b}(\tau)$ and
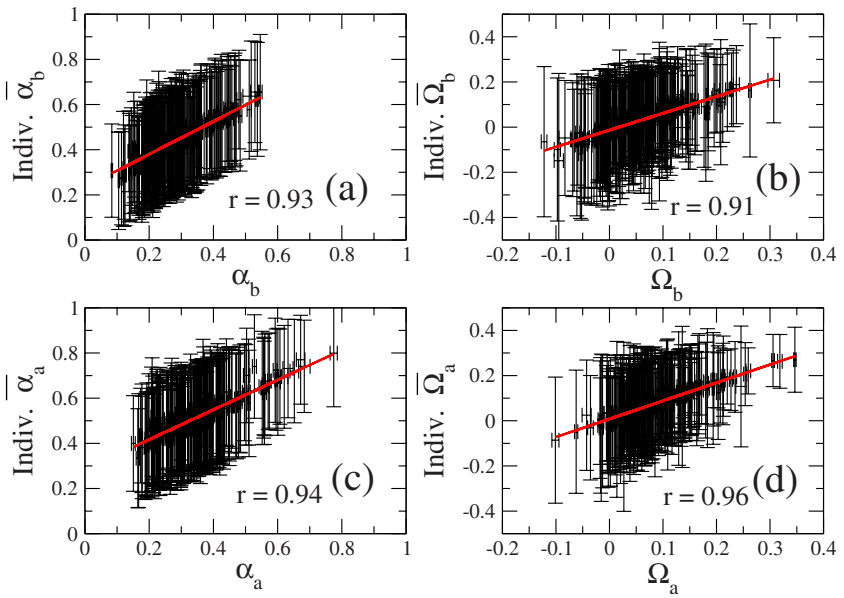

FIG. 7. (Color online) In order to account for the dispersion in the pdf's plotted in Figs. 6(c) and 6(d) for individual stocks, we compare the average values $\bar{\alpha}_{a, b}$ and $\bar{\Omega}_{a, b}$ computed from all $N_{a, b}^{j}(\tau)$ with $\alpha_{a, b}$ and $\Omega_{a, b}$ computed from the corresponding average market response $N_{a, b}(\tau)$ for each of the $219 T_{c}$ 's. The visually apparent correlation indicates that the parameters quantifying $N_{a, b}(\tau)$ are a good representation of the average $N_{a, b}^{j}(\tau)$. The correlation coefficient $r$ for each linear regression is provided in each panel.

$N_{a}(\tau)$ corresponding to Eq. (6), while Figs. 6(c) and 6(d) show the distribution of parameter values calculated from the individual stock responses $N_{b}^{j}(\tau)$ and $N_{a}^{j}(\tau)$. The pdf's for individual stock values of $\Omega$ and $\alpha$ have a larger dispersion, as the response to each market shock is not uniform across all stocks. For the average market response $N(\tau)$ in Figs. 6(a) and 6(b) the pdf's of $\Omega$ and $\alpha$ are shifted to larger values for $t>T_{c}$ as compared to $t<T_{c}$. This is indicative of the stress that can build prior to anticipated announcements [42] and the surprise that is inherent in the news. Larger $\Omega$ values correspond to faster relaxation times, while larger $\alpha$ values correspond to higher activity. We also observe $\Omega<0$, which corresponds to particular time series in which the preshocks or aftershocks farther away from the main shock (for large $\tau$ ) are dominant over the volatility cascade around $T_{c}$. The values of the Omori parameters we find on averaging over all market shocks are given in the figure caption.

Although there is a wide distribution of Omori parameter values when considering all 219 market shocks, there is a strong correlation between the individual stock dynamics for a given market shock. In Fig. 7 we relate the values of $\alpha$ and $\Omega$ calculated for the average market response to the average and standard deviation of $\alpha$ and $\Omega$ calculated for individual stocks for a given $T_{c}$. The strong correlation between these quantities over 219 different dates indicates that the dispersion in the values of $\alpha$ and $\Omega$ for individual stocks, as demonstrated in Figs. 6(a) and 6(c), results from the broad range of $V\left(T_{c}\right)$ values and, further, that the dispersion does not result merely from the range of stocks analyzed.

In Fig. 8 we plot the relation between the magnitude $M$ of each main shock and the resulting Omori exponents $\Omega_{a, b}$ calculated from both market $N_{a, b}(\tau)$ and individual stock $N_{a, b}^{j}(\tau)$ response curves. Figures 8(a) and 8(c) show a positive relation between $M$ and the decay exponent $\Omega_{a}$, which indicates that the market responds faster to large shocks on 

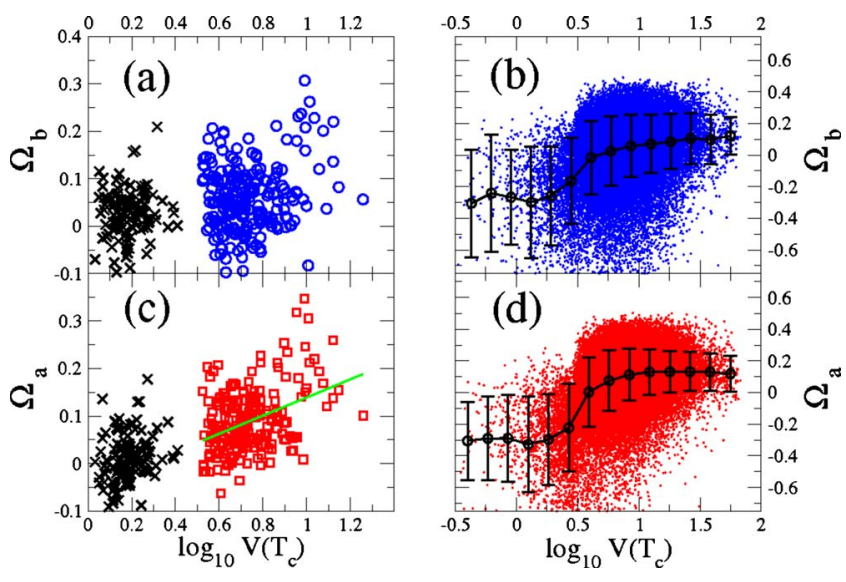

FIG. 8. (Color online) The relation between the magnitude $M \equiv \log _{10} V\left(T_{c}\right)$ and the Omori exponents $\Omega_{a, b}$. In (a) and (c) we compare values calculated from the average market response $N_{a, b}(\tau)$, and in (b) and (d) we compare values calculated from individual stock response $N_{a, b}^{j}(\tau)$. (a) Weak relation before $T_{c}$, where we validate the linear regression model at $p=0.001$ significance level, but with correlation coefficient $r=0.22$. The dispersion may result from the variability in anticipation preceding the market shock at $T_{c}$. (c) The relation between $\Omega_{a}$ and $M$ is stronger after $T_{c}$ than before $T_{c}$, with linear regression significance $p \approx 0$, correlation $r=0.40$, and regression slope $m=0.19 \pm 0.03$. The increasing trend demonstrates that a faster response, quantified by larger $\Omega_{a}$, follows a larger $M$. Data points in (a) and (c) denoted by the symbol $\times$ correspond to values of $\Omega_{a, b}$ calculated for randomly selected $T_{c}$ on those 118 days analyzed without a single value of $x(t)>x_{c}$. In (b) and (d) there is much dispersion in the $\Omega$ values of individual stocks for given $V\left(T_{c}\right)$. However, the average trends demonstrate a significant crossover at $M_{\mathrm{x}} \approx 0.5$ from $\Omega_{a, b}<0$ to $\Omega_{a, b}>0$. The case of $\Omega<0$ can occur when there is more volatility clustering for large $\tau$ than for small $\tau$, whereas the case of $\Omega>0$ occurs for large volatility cascading around $\tau \gtrsim 0$. This crossover could result from the difference between anticipated and surprise shocks at $T_{c}$.

the intraday time scale. Figures 8(b) and 8(d) show a significant dispersion across all stocks for a given date. Interestingly, we find a crossover at $M_{\mathrm{x}} \approx 0.5$ above which $\Omega_{a, b}$ increases sharply to positive values. The values $\Omega \approx 0$ for $M$ $<M_{\mathrm{x}}$ correspond to a dynamical cascade $n(\tau)$ that is indistinguishable from an exponential decay. Typically, small values of $\Omega$ correspond to stocks with relatively low trading activity, which are less sensitive to market shocks. For individual stocks, we define $M$ to be the logarithm of the largest volatility within $3 \mathrm{~min}$ of the main shock $T_{c}$ measured for the average market response $N(\tau)$. This accounts for the possibility of a stock-specific anticipation or delay time in the volatility as a result of the main shock $V\left(T_{c}\right)$. There is also the possibility that a spurious value of $\Omega \approx 0$ can arise from a stock which has a constant (potentially large) level of activity throughout the entire time period analyzed.

In Fig. 9 we plot the relation between the magnitude $M$ and the Omori-law amplitude $\alpha_{a, b}$ for both market $N_{a, b}(\tau)$ and individual $N_{a, b}^{j}(\tau)$ response curves. The relation between $\alpha$ and $M$ is stronger than the relation between $\Omega$ and $M$, indicating that the Omori-law amplitude has a higher information content than the Omori-law exponent. The strong relation for the average market response suggests that it is
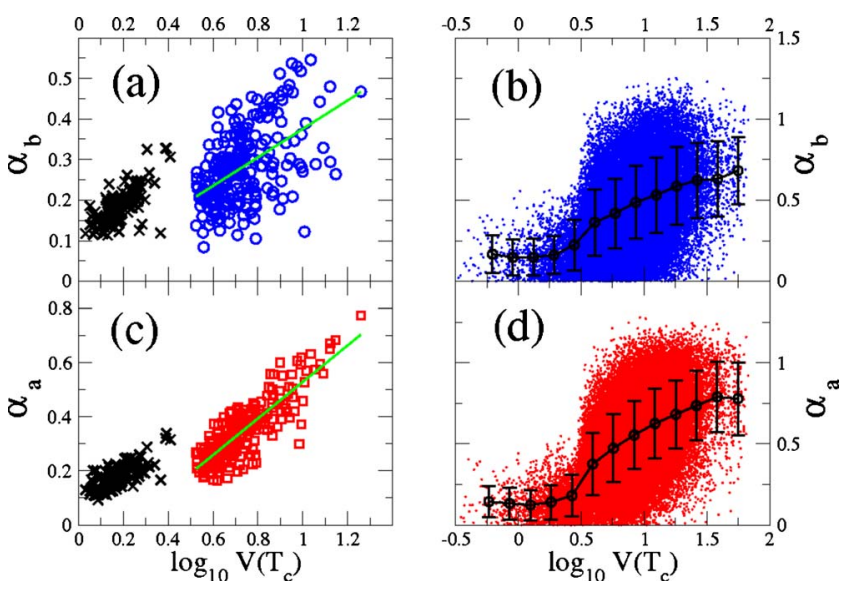

FIG. 9. (Color online) The relation between the magnitude $M \equiv \log _{10} V\left(T_{c}\right)$ and the Omori amplitudes $\alpha_{a, b}$. In (a) and (c) we compare the values calculated from the average market response $N(\tau)$, and in (b) and (d) we compare values calculated from individual stock response $N_{a, b}^{j}(\tau)$. (a) The increasing relation between $\alpha_{b}$ and $M$ is statistically stronger than the relation between $\Omega_{b}$ and $M$ in Fig. 8(a), with significance $p \approx 0$, correlation coefficient $r=0.52$, and regression slope $m=0.35 \pm 0.04$. (c) The relation between $\alpha_{a}$ and $M$ is strong, with significance $p \approx 0, r=0.84$, and regression slope $m=0.68 \pm 0.03$. Data points in (a) and (c) denoted by the symbol $\times$ correspond to values of $\alpha_{a, b}$ calculated for randomly selected $T_{c}$ on those 118 days analyzed without a single value of $x(t)>x_{c}$. The result that $\alpha$ increases with increasing $V\left(T_{c}\right)$ holds even for random times. In (b) and (d) there is much dispersion in the $\alpha$ values of individual stocks for given $V\left(T_{c}\right)$. However, the average trends demonstrate a significant crossover at $M_{\mathrm{x}} \approx 0.5$ from $\alpha_{a, b} \approx 0.2$ for $M<0.5$ to $\alpha_{a, b}>0.2$ for $M>0.5$. This crossover occurs at a similar location as the crossover observed in Figs. 8(b) and $8(\mathrm{~d})$ for $\Omega_{b, a}$. The average amplitude value $\bar{\alpha}$ increases sharply for $M>M_{\mathrm{x}}$, consistent with first-order phase-transition behavior.

possible to identify precursors of market shocks with statistical certainty. However, since often $T_{c}$ corresponds to anticipated market news, the significant activity prior to the main shock is a natural by-product of trader anticipation. Interestingly, we also observe a critical threshold for $M_{\mathrm{x}} \approx 0.5$, above which the average response amplitude $\alpha_{a, b}$ increases suddenly, analogous to a first-order transition.

Behaviors of the market cascades above and below $M_{\mathrm{x}}$ are significantly different. Below $M_{\mathrm{x}}$, it is typical for $\Omega_{a, b}$ to be negative and $\alpha_{a, b} \approx 0$, indicating that the triggering shock $V\left(T_{c}\right)$ is relatively insignificant, with relatively few preshocks and aftershocks. In this scenario, it is possible for a few clusters of relatively large volatility toward the end of the time series $N_{a, b}^{j}(\tau)$ to dominate in the calculation of the best-fit parameters, producing negative values for $\Omega_{a, b}$. There are also cases for both $N_{a, b}^{j}(\tau)$ and $N_{a, b}(\tau)$ for which $\Omega_{a, b} \approx 0$, corresponding to a constant rate of preshocks and aftershocks in the time period analyzed.

However, above $M_{\mathrm{x}}$, the cascade around $T_{c}$ is more significant, but with some anomalous properties. Namely, in the case of the market response $N_{a, b}(\tau)$, there is an increasing relation between $M$ and $\Omega_{a}$, indicating that the market responds more quickly to larger market shocks. This observation is consistent with the "semistrong" efficient-market hy- 


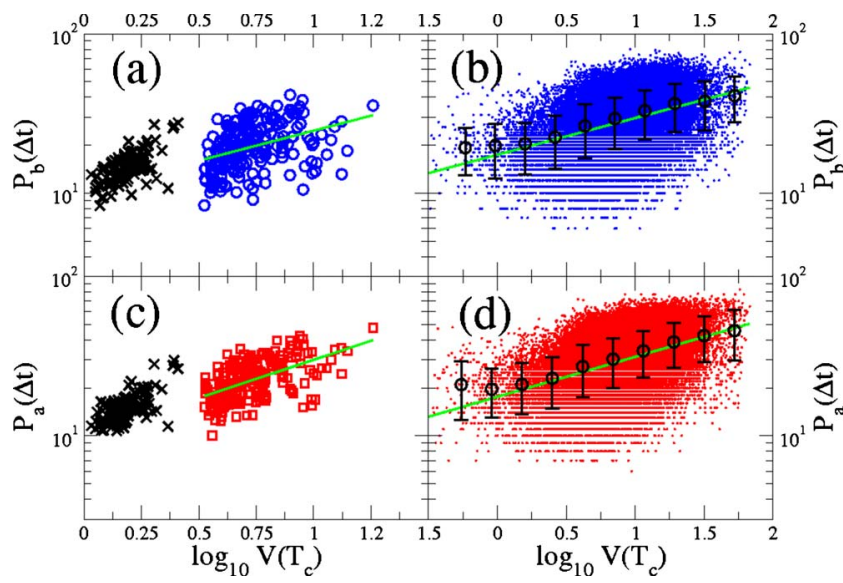

FIG. 10. (Color online) The increasing relation between the productivity $P_{a, b}(\Delta t)$ of each market shock and the size of the main shock $M \equiv \log _{10} V\left(T_{c}\right)$ with $\Delta t \equiv 90 \mathrm{~min}$. As is found in earthquakes, we find a power-law relationship between $M$ and $V\left(T_{c}\right)$ described by a productivity exponent $\Pi_{b}$ before and exponent $\Pi_{a}$ after the market shock. Data points in (a) and (c) denoted by the symbol $\times$ correspond to values of $P_{a, b}(\Delta t)$ calculated for randomly selected $T_{c}$ on those 118 days analyzed without a single value of $x(t)>x_{c}$. The result that $P(\Delta t)$ increases with increasing $V\left(T_{c}\right)$ holds even for random times. For the average market response $N_{b, a}(\Delta t)$, we find (a) $\Pi_{b}=0.38 \pm 0.07$ and (c) $\Pi_{a}=0.48 \pm 0.04$. For the productivity of individual stocks corresponding to $N_{b, a}^{j}(\Delta t)$ we find (b) $\Pi_{b}=0.23 \pm 0.01$ and (d) $\Pi_{a}=0.25 \pm 0.01$. For comparison, the power-law exponent value pertaining to earthquake aftershocks is $\Pi_{a} \approx 0.7-0.9[38]$.

pothesis, which asserts that markets incorporate public news approximately instantly. This observation is similar to geophysical earthquakes, where it is observed that the value of $\Omega_{a}$ increases with $M$ for a given geographical location [43]. Also, for geophysical earthquakes, it is also found that the Omori amplitude increases exponentially with $M$ [38]. In the case of individual company response $N_{a, b}^{j}(\tau)$, the values of the average $\Omega_{a, b} \approx 0.1$ saturate above $M_{\mathrm{x}}$, whereas the values of $\alpha_{a, b}$ increase with $M$. Thus, the stochastic dynamics display a nonlinear relationship with $M$, consistent with a nonlinear shot noise process [44], and are a potential avenue for future theoretical research.

In Fig. 10 we plot the relation between $V\left(T_{c}\right)$ and the productivity $P_{a}(\Delta t)$ [or $\left.P_{b}(\Delta t)\right]$, defined as the cumulative number of aftershocks (or preshocks) greater than the threshold $q \equiv 3$ within $\Delta t \equiv 90 \min$ of $T_{c}$. Motivated by the powerlaw relationship observed for earthquakes we fit the relations $P_{a}(\Delta t) \sim M^{\Pi_{a}}$ and $P_{b}(\Delta t) \sim M^{\Pi_{b}}$ and find statistically significant values for the market response $\Pi_{b}=0.38 \pm 0.07$ and $\Pi_{a}=0.48 \pm 0.04$, and for individual stocks $\Pi_{b}=0.23 \pm 0.01$ and $\Pi_{a}=0.25 \pm 0.01$. For earthquakes, Ref. [38] reports a range of $\Pi_{a} \approx 0.7-0.9$ values that are larger than those observed here for financial markets, meaning that the productivity of physical earthquakes increases "faster" with main shock magnitude than does the productivity of market shocks. Since for earthquakes $\Pi_{a}<b$, where $b \approx 1$ is the scaling exponent in the Gutenberg-Richter law, this inequality establishes the relative importance of small fluctuations as compared to large fluctuations [38]. In other words, this
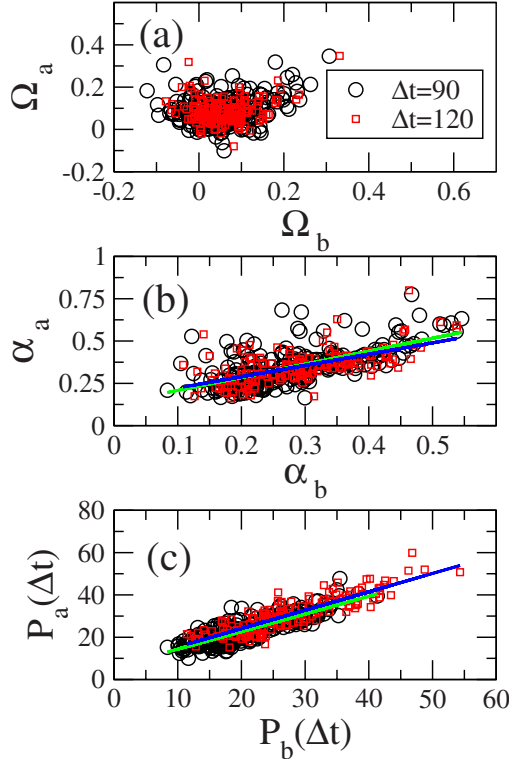

FIG. 11. (Color online) A comparison of Omori parameters before and after $T_{c}$ for $N(\tau)$ and varying $\Delta t$ indicate that $\alpha_{b}$ and $P_{b}(\Delta t)$ are better conditional estimators for the dynamics after $T_{c}$. (a) Weak relationship between $\Omega_{b}$ and $\Omega_{a}$ for $\Delta t=90$ and 120. (b) Strong relationship between $\alpha_{b}$ and $\alpha_{a}$ for $\Delta t=90$ and 120, with both linear regressions passing the ANOVA (analysis of variance) $\mathrm{F}$ test at the $p<0.001$ confidence level. (c) Strong relationship between $P_{b}(\Delta t)$ and $P_{a}(\Delta t)$ for $\Delta t=90$ and $120 \mathrm{~min}$ at the $p<0.001$ confidence level.

inequality indicates that small earthquakes play a larger role than large earthquakes in producing the observed number of large earthquake shocks. Using an analogous argument for market volatility, since the cumulative distribution exponent $\eta_{V} \approx 3$ is found to be robust across many markets [33,34], then the total number $N_{T o t}(V)$ of aftershocks triggered by all main shocks of size $V$ scales as

$$
N_{\text {Tot }}(V)=P(V) P_{a}(\Delta t) \sim 10^{\left(\Pi_{a}-\eta_{V}\right) \log _{10} V},
$$

which is a decreasing function of $V$. Hence, we also find that aftershock cascade triggering is controlled by the contributions of the more numerous small shocks. Thus, the mediumsized market shocks (analyzed here) play a larger role than the large market shocks in producing the observed heavytailed distribution of market shocks. We further note that the productivity is a combination of the relationships of both $\alpha$ and $\Omega$ with $V\left(T_{c}\right)$, which can be written as

$$
P_{a}(\Delta t) \equiv N_{a}(\Delta t) \sim(\Delta t)^{1-\Omega_{a}} \alpha_{a} /\left(1-\Omega_{a}\right) \sim V\left(T_{c}\right)^{\Pi_{a}},
$$

with equivalent relation before the shock for $P_{b}(\Delta t)$.

In Fig. 11 we plot the values $\Omega, \alpha$, and $P(\Delta t)$ both before and after the main shock at time $T_{c}$. Surprisingly, while there is little statistical relation between $\Omega_{b}$ and $\Omega_{a}$, there is a strong relation between $\alpha_{b}$ and $\alpha_{a}$ as well as between $P_{b}(\Delta t)$ and $P_{a}(\Delta t)$ for both $\Delta t=90$ and $\Delta t=120 \mathrm{~min}$. This result could be of interest for volatility and option traders who would like to anticipate the market dynamics after an announcement, given the dynamics before the announcement. 

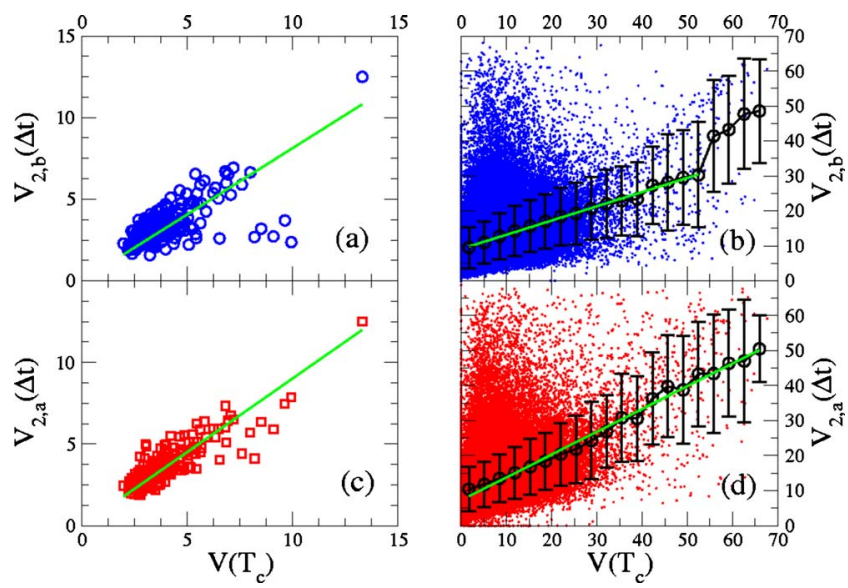

FIG. 12. (Color online) The increasing relation between the size of the main shock $V\left(T_{c}\right)$ and the size of the second largest aftershock (or preshock) $V_{2}(\Delta t)$ within $\Delta t$ minutes of $T_{c}$ demonstrate that the volatility of the largest aftershock (or preshock) increases with main shock volatility. As with the Bath law for earthquakes, we observe a proportional relation $V_{2, a}(\Delta t) \equiv C_{B} V\left(T_{c}\right)$, which corresponds to a Bath parameter $B=-\log _{10} C_{B}$. For the average market response $N_{b, a}(\Delta t)$ we calculate $C_{B}$ for (a) the dynamics before, $C_{B}=0.81$, with correlation coefficient $r=0.70$ and $\chi^{2}=212$, and for (c) the dynamics after, $C_{B}=0.9$, with $r=0.87$ and $\chi^{2}=109$. For the Bath law corresponding to individual stocks we find that a linear function best fits the relation between $V\left(T_{c}\right)$ and the average value $\bar{V}_{2}(\Delta t)$ calculated for equal-sized bins as indicated by circles with one standard deviation error bars. We calculate that the regression slope for the Bath law (b) before is $m=0.65 \pm 0.02$ and (d) after is $m=0.40 \pm 0.01$.

In Fig. 12 we relate the size of the largest shock $V_{1}$ $\equiv V\left(T_{c}\right)$ to the sizes of the second largest shock $V_{2}$ both before and after $T_{c}$. The Bath law parameter $B$ quantifies the relation between $V_{1}$ and $V_{2}$ as

$$
M_{1}-M_{2}=\log _{10} V_{1}-\log _{10} V_{2}=B \text {. }
$$

This functional form implies the relation

$$
V_{2} / V_{1}=C_{B}
$$

and hence $B=-\log _{10} C_{B}$. Figure $12(\mathrm{c})$ is a scatter plot of $V_{1}$ and $V_{2, a}$ which shows a linear relation corresponding to $B_{a}$ $=-\log _{10}(0.90)=0.046$. Surprisingly, Fig. 12(a) also shows a strong relation between $V_{1}$ and $V_{2, b}$ with $B_{b}=-\log _{10}(0.81)$ $=0.092$. Comparing the values of $B_{b}$ and $B_{a}$, the difference between $V_{1}$ and $V_{2}$ is smaller after $T_{c}$ than before $T_{c}$. Interestingly, both $B_{b}$ and $B_{a}$ are significantly smaller than the value $B_{E} \approx 1.2$ observed for earthquake aftershocks [37], meaning that the largest preshock and aftershock are of comparable magnitude to the main shock. This significant difference between earthquakes and market shocks is largely due to the relative probabilities of observing first- and secondlargest events $x_{1}$ and $x_{2}$. The conditional probability $P\left(x_{1} \mid x_{2}\right)=P\left(x_{1}>x_{2}\right)$ is given by the corresponding cumulative distribution function. Hence, using Eqs. (9) and (10), the ratio of the conditional probabilities for $E_{1}$ and $V_{1}$ is
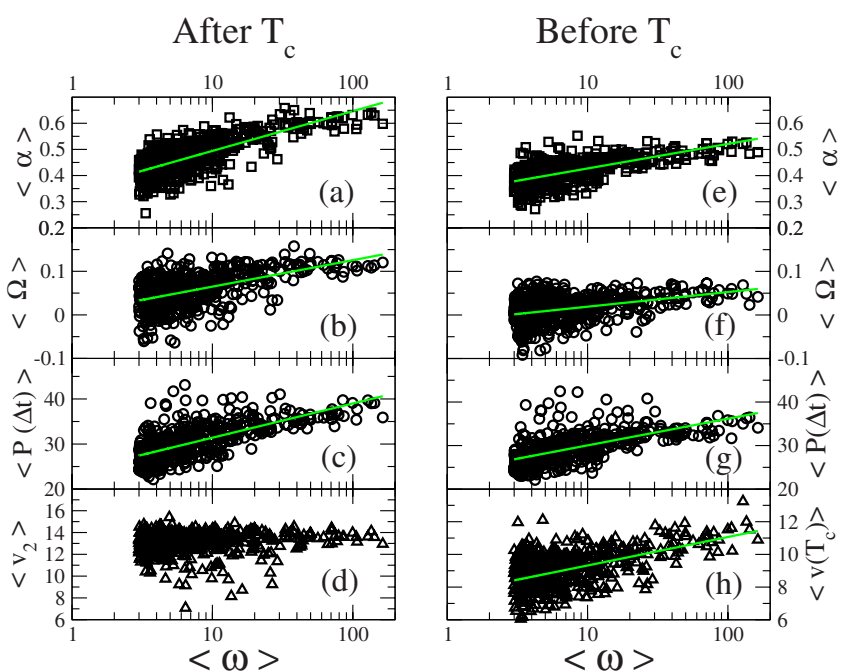

FIG. 13. (Color online) Relations between individual stock trading activity and dynamic response parameters (a)-(d) after $T_{c}$ and (e)-(h) before $T_{c}$, averaged over all the days with a market shock. We measure the trading activity $\langle\omega\rangle$ for each stock, defined as the average number of transactions per minute over the $2 \mathrm{yr}$ period of 2001-2002. We find that stocks with large trading activity react both more strongly [larger $\alpha$ and larger $P(\Delta t)$ ] and quickly (larger $\Omega$ ) to market shocks. However, (d) shows that there is little relation between $\langle\omega\rangle$ and the average size of the largest aftershock $\left\langle v_{2}\right\rangle$.

$$
\frac{P\left(V_{1} \mid V_{2}\right)}{P\left(E_{1} \mid E_{2}\right)}=\frac{P\left(V_{1}>V_{2}\right)}{P\left(E_{1}>E_{2}\right)} \sim \frac{V_{2}^{-3}}{E_{2}^{-2 / 3}},
$$

which roughly explains the $10^{2}$ factor difference $B_{E}$ $\approx 10^{2} B_{V}$

We also compare the volatilities $V_{1}$ and $V_{2}$ for individual stocks in Fig. 12(b) before $T_{c}$ and in Fig. 12(d) after $T_{c}$. We compute the average value $\left\langle V_{2}\right\rangle$ for linear bins and find $V_{1}$ $>\left\langle V_{2}\right\rangle$ for $V_{1}>20$ both before and after $T_{c}$. Also, Fig. 12 shows that $\left.\left\langle V_{2, a}\right\rangle\right\rangle\left\langle V_{2, b}\right\rangle$ for most values of $V\left(T_{c}\right)$. Hence, the reaction to surprise causes larger volatility fluctuations than the anticipation of surprise.

We further ask the question of how do the response parameters analyzed here depend on the variations between individual stock trading patterns. To answer this question, we quantify the trading capacity of each stock by $\langle\omega\rangle$, the average number of transactions per minute, with $3 \leq\langle\omega\rangle \leq 163$ for the $S=531$ stocks analyzed. We hypothesize that $\langle\omega\rangle$ is closely related to firm size and market impact. Figure 13(a) shows $\langle\alpha\rangle,\langle\omega\rangle$, and $\langle P(\Delta t)\rangle$ increase with $\langle\omega\rangle$ after $T_{c}$, indicating that stocks with a large trading base respond to market shocks with large volatility $\left\langle v\left(T_{c}\right)\right\rangle$ [shown in Fig. 13(b)], but also relax more quickly, corresponding to larger $\Omega$ values. However, we find no statistical relation between $\langle\omega\rangle$ and $\left\langle v_{2, a}\right\rangle$. Interestingly, Fig. 13(b) shows that this positive relation also applies to the dynamic response parameters before $T_{c}$.

\section{DISCUSSION}

Cascading avalanche dynamics are a common phenomena in complex systems ranging in scale from solar flares $[35,36]$ 
and earthquakes $[10,11,39,40]$ to microscopic vortices in turbulent fluids [45]. Similar bursting phenomena is also observed in human organs, such as the heart [46,47], lungs $[48,49]$, and brain [50-52], and also for common social [53-56] and economic [12-15,22,23,57,58] systems. Neural avalanches in the brain are frequent even in the resting state and are a signature of healthy brain functioning within the neural network. In fact, the ability to process and disseminate information is largely attributed to the network structure of neuronal correlations which, if inhibited by disease, lead to altered dysfunctional states such as in the case of schizophrenia. Extending by analogy, the frequency of cascades in financial markets could also be viewed as a "healthy" optimal state for processing information and eliminating arbitrage among the many the degrees of freedom. Recent work [59] on the switching dynamics around highs and lows in financial time series shows further evidence of Omori power-law scaling before and after microtrend extrema, in analogy to the market shocks at $T_{c}$ developed here. Interestingly, this work on switching dynamics finds cascading trends on time scales ranging from seconds to hundreds of days.

Financial markets are subject to constant information flow, resulting in a large rate of significant events, such as Fed announcements $[15,42]$, quarterly earnings, splits and dividends announcements, mergers and acquisitions, and institutional reports. This information can arrive as "expected" or come as a "surprise." Interestingly, there are precursors extending more than 1 day in advance of expected announcements such as earnings announcements [57]. Economists have long been interested in the interplay between informed and uninformed traders and in the dissemination of information across a market consisting of rational agents. Early work focuses on the relationship between trading volume and price change and the relationship between these quantities and the qualitative notions of surprise, importance, and precision of the information [58].

Using methods from statistical physics $[4,5,60]$ and geophysics, we analyze the absolute returns of price because of the long-memory property and the universality of fluctuations in this quantity across diverse markets [33,34,61,62]. Reference [58] postulates that price changes reflect the average change in market expectations, whereas trading volume reflects idiosyncratic reactions across all traders. Recent work further quantifies trading volume fluctuations and finds that they are similar to price fluctuations and, furthermore, finds significant cross correlation between volume change and price change [63]. Omori relaxation dynamics are also shown for trading volume in [15]. Here, we also observe significant volume cascading as evident in Fig. 4. The analysis of volume and transaction dynamics is an avenue of future research and could highlight the relationship between volume and price fluctuations by studying their correlation around market shocks.

To summarize, we analyzed the cascade dynamics of price volatility, which has potential applications in options pricing and the pricing of other derivatives. The Black-Scholes equation in its simple form assumes that the fluctuations in the price are constant during the duration of the option [64]. However, more sophisticated methods [65] incorporate timedependent price volatility and are more realistic descriptions of the nonstationarity of financial time series. The results in this paper are of potential interest for traders modeling derivatives on short time scales around expected market shocks, e.g., earnings reports. The statistical regularity of both market and individual stock behaviors before and after a market shock of magnitude $M \equiv \log _{10} V\left(T_{c}\right)$ provides information that could be used in hedging, since we observe a crossover in the cascade dynamics for $M \approx 0.5$. Knowledge of the Omori response dynamics provides a time window over which aftershocks can be expected. Similarly, the productivity law provides a more quantitative value for the number of aftershocks to expect. Finally, the Bath law provides conditional expectation of the largest aftershock and even the largest preshock, given the size of the main shock. Of particular importance, from the inequality of the productivity law scaling exponents and the pdf scaling exponent for price volatility, we find that the role of small fluctuations is larger than the role of extremely large fluctuations in accounting for the prevalence of aftershocks.

\section{ACKNOWLEDGMENTS}

We thank L. de Arcangelis for the idea of investigating the Bath and productivity laws; K. Yamasaki and A. Ralph for helpful suggestions; and NSF, DTRA, and ONR for financial support.
[1] E. F. Fama, J. Business 38, 34 (1965).

[2] Z. Ding, C. W. J. Granger, and R. F. Engle, J. Empirical Finance 1, 83 (1993).

[3] B. Mandelbrot, J. Business 36, 394 (1963).

[4] R. N. Mantegna and H. E. Stanley, Econophysics: An Introduction (Cambridge University Press, Cambridge, England, 1999).

[5] J. P. Bouchaud and M. Potters, Theory of Financial Risk (Cambridge University Press, Cambridge, England, 2000).

[6] J. P. Bouchaud, Quant. Finance 1, 105 (2001).

[7] X. Gabaix, P. Gopikrishnan, V. Plerou, and H. E. Stanley, Nature (London) 423, 267 (2003).
[8] J. D. Farmer, M. Shubik, and E. Smith, Phys. Today 58(9), 37 (2005).

[9] X. Gabaix, P. Gopikrishnan, V. Plerou, and H. E. Stanley, Q. J. Econ. 121, 461 (2006).

[10] F. Omori, J. Coll. Sci., Imp. Univ. Tokyo 7, 111 (1894).

[11] T. Utsu, Geophys. Mag. 30, 521 (1961).

[12] S. Ghashghaie, W. Breymann, J. Peinke, P. Talkner, and Y. Dodge, Nature (London) 381, 767 (1996).

[13] F. Lillo and R. N. Mantegna, Phys. Rev. E 68, 016119 (2003).

[14] P. Weber, F. Wang, I. Vodenska-Chitkushev, S. Havlin, and H. E. Stanley, Phys. Rev. E 76, 016109 (2007).

[15] A. M. Petersen, F. Wang, S. Havlin, and H. E. Stanley, Phys. 
Rev. E 81, 066121 (2010)

[16] J. D. Hamilton and O. Jorda, J. Polit. Econ. 110, 1135 (2002).

[17] J. D. Hamilton, J. Money, Credit Banking 41, 567 (2009).

[18] D. Sornette and A. Helmstetter, Physica A 318, 577 (2003).

[19] D. Sornette, Y. Malevergne, and J. F. Muzy, Risk 16, 67 (2003).

[20] New York Times article archive, 1981-present, http:// www.nytimes.com/ref/membercenter/nytarchive.html

[21] A. G. Zawadowski, G. Andor, and J. Kertész, Quant. Finance 6, 283 (2006).

[22] A. Joulin, A. Lefevre, D. Grunberg, and J. P. Bouchaud, Wilmott Magazine 46, 1 (2008); e-print arXiv:0803.1769.

[23] A. Ponzi, F. Lillo, and R. N. Mantegna, Phys. Rev. E 80, 016112 (2009).

[24] F. Wang, S.-J. Shieh, S. Havlin, and H. E. Stanley, Phys. Rev. E 79, 056109 (2009).

[25] K. Yamasaki, L. Muchnik, S. Havlin, A. Bunde, and H. E. Stanley, Proc. Natl. Acad. Sci. U.S.A. 102, 9424 (2005).

[26] F. Wang, K. Yamasaki, S. Havlin, and H. E. Stanley, Phys. Rev. E 73, 026117 (2006)

[27] F. Wang, P. Weber, K. Yamasaki, S. Havlin, and H. E. Stanley, Eur. Phys. J. B 55, 123 (2007).

[28] R. N. Mantegna, Eur. Phys. J. B 11, 193 (1999).

[29] L. Laloux, P. Cizeau, J. P. Bouchaud, and M. Potters, Phys. Rev. Lett. 83, 1467 (1999).

[30] V. Plerou, P. Gopikrishnan, B. Rosenow, L. A. Nunes Amaral, and H. E. Stanley, Phys. Rev. Lett. 83, 1471 (1999).

[31] V. Plerou, P. Gopikrishnan, B. Rosenow, Luis A. Nunes Amaral, T. Guhr, and H. E. Stanley, Phys. Rev. E 65, 066126 (2002).

[32] Historical Data for Key Federal Reserve Interest Rates, http:// www.federalreserve.gov/releases/h15/data.htm; http:// www.federalreserve.gov/fomc/fundsrate.htm

[33] V. Plerou, P. Gopikrishnan, L. A. Nunes Amaral, M. Meyer, and H. E. Stanley, Phys. Rev. E 60, 6519 (1999).

[34] P. Gopikrishnan, V. Plerou, L. A. Nunes Amaral, M. Meyer, and H. E. Stanley, Phys. Rev. E 60, 5305 (1999).

[35] L. de Arcangelis, C. Godano, E. Lippiello, and M. Nicodemi, Phys. Rev. Lett. 96, 051102 (2006).

[36] L. de Arcangelis, E. Lippiello, C. Godano, and M. Nicodemi, Eur. Phys. J. B 64, 551 (2008).

[37] G. Drakatos and J. Latoussakis, J. Seismol. 5, 137 (2001).

[38] A. Helmstetter, Phys. Rev. Lett. 91, 058501 (2003).

[39] A. Saichev and D. Sornette, Tectonophysics 431, 7 (2007).

[40] A. Saichev and D. Sornette, Phys. Rev. E 70, 046123 (2004).

[41] M. Bottiglieri, L. de Arcangelis, C. Godano, and E. Lippiello, Phys. Rev. Lett. 104, 158501 (2010).
[42] S. Demiralp and O. Jordá, Federal Reserve Bank of New York, Economic Policy Review 8(1), pp. 29-48 (2002).

[43] G. Ouillon and D. Sornette, J. Geophys. Res. 110, B04306 (2005).

[44] I. Eliazar and J. Klafter, Proc. Natl. Acad. Sci. U.S.A. 102 13779 (2005).

[45] A. N. Kolmogorov, Proc. R. Soc. London, Ser. A 434, 9 (1991).

[46] P. Ch. Ivanov, L. A. N. Amaral, A. L. Goldberger, S. Havlin, M. G. Rosenblum, Z. Struzik, and H. E. Stanley, Nature (London) 399, 461 (1999).

[47] D. C. Lin and R. L. Hughson, Phys. Rev. Lett. 86, 1650 (2001).

[48] B. Suki, A.-L. Barabasi, Z. Hantos, F. Petak, and H. E. Stanley, Nature (London) 368, 615 (1994).

[49] A. M. Alencar, S. V. Buldyrev, A. Majumdar, H. E. Stanley, and B. Suki, Phys. Rev. Lett. 87, 088101 (2001).

[50] T. Petermann, T. C. Thiagarajan, M. A. Lebedev, M. A. L. Nicolelis, and D. R. Chialvo, Proc. Natl. Acad. Sci. U.S.A. 106, 15921 (2009).

[51] D. Plenz and D. R. Chialvo, e-print arXiv:0912.5369.

[52] J. Touboul and A. Destexhe, PLoS ONE 5, e8982 (2010).

[53] R. Crane and D. Sornette, Proc. Natl. Acad. Sci. U.S.A. 105, 15649 (2008).

[54] A. Vázquez, J. Gama Oliveira, Z. Dezsö, K.-I. Goh, I. Kondor, and A.-L. Barabási, Phys. Rev. E 73, 036127 (2006).

[55] K.-L. Goh and A.-L. Barabási, EPL 81, 48002 (2008).

[56] D. Rybski, S. Buldyrev, S. Havlin, F. Liljeros, and H. A. Makse, Proc. Natl. Acad. Sci. U.S.A. 106, 12640 (2009).

[57] D. Morse, J. Account. Res. 19, 374 (1981).

[58] O. Kim and R. E. Verrecchia, J. Account. Res. 29, 302 (1991).

[59] T. Preis and H. E. Stanley, J. Stat. Phys. 138, 431 (2010).

[60] R. Cont, M. Potters, and J. P. Bouchaud, Proceedings of the Les Houches Workshop, Les Houches, France, 1997 (Springer, Berlin, 1997), pp. 1-11.

[61] Y. Liu, P. Cizeau, M. Meyer, C.-K. Peng, and H. E. Stanley, Physica A 245, 437 (1997); P. Cizeau, Y. Liu, M. Meyer, C.-K. Peng, and H. E. Stanley, ibid. 245, 441 (1997).

[62] Y. Liu, P. Gopikrishnan, P. Cizeau, M. Meyer, C.-K. Peng, and H. E. Stanley, Phys. Rev. E 60, 1390 (1999).

[63] B. Podobnik, D. Horvatic, A. M. Petersen, and H. E. Stanley, Proc. Natl. Acad. Sci. U.S.A. 106, 22079 (2009).

[64] B. E. Baaquie, Quantum Finance: Path Integrals and Hamiltonians for Options and Interest Rates (Cambridge University Press, Cambridge, UK, 2004).

[65] J. P. Bouchaud and D. Sornette, J. Phys. I 4, 863 (1994). 